\title{
Management of painful reverse shoulder arthroplasty
}

Shoulder \& Elbow

2017, Vol. 9(3) 212-222

(C) The Author(s) 2017

Reprints and permissions:

sagepub.co.uk/journalsPermissions.nav

DOI: 10.1 I77/1758573217702333

journals.sagepub.com/home/sel

SAGE

\section{Anders L. Ekelund}

\begin{abstract}
Even though reverse shoulder arthroplasty is a very successful procedure, painful complications occur. During the initial postoperative years, the most common reasons for pain are instability, postoperative fracture of the acromion or spine, and periprosthetic infection. Later, aseptic loosening, with humeral loosening being more frequent that glenoid loosening, can be a source of pain and reduction in function. A careful patient history, clinical examination, plain radiographs, computed tomography and blood tests give an explanation for the pain in most cases. The majority of these complications can be successfully treated, maintaining a functional reverse shoulder arthroplasty. However, if all examinations are normal, it is important to remember that nonshoulder conditions such as tumour of the lung or degenerative changes of the cervical spine can give shoulder pain.
\end{abstract}

Keywords

infection, instability, pain, reverse arthroplasty, scapular spine fracture

Date received: 24th February 20I7; accepted: 2nd March 2017

\section{Introduction}

The indications for reverse shoulder arthroplasty (RSA) have expanded over the last 20 years and, in some centres, more reverse arthroplasties than anatomical shoulder replacements are performed. Table 1 illustrates my indications for RSA. The majority of patients scheduled for a RSA have significant pain, even though the procedure is sometimes performed just to restore function in pseudoparalytic shoulders. Because more reverse arthroplasties are being carried out, the number of patients with a complication is increasing. Some of these complications are painful. The majority of patients who are dissatisfied with the procedure complain of pain.

Here, a review of how to approach a painful reverse arthroplasty is provided, based on published reports and 20 years of personal experience of the RSA.

\section{Postoperative recovery}

It is normal to have pain several months after surgery. ${ }^{1,2}$ There is a huge individual variation in the duration and intensity of postoperative pain. Levy et al. ${ }^{1}$ found that shoulder function improved during the first postoperative year after an RSA. Pain, measured by visual analogue scale, decreased postoperatively and $85 \%$ of the improvement was seen at 3 months. Simovitch et al. ${ }^{2}$ found that most of the improvement after RSA ocurred during the first 6 months after surgery, and there was maximum improvement after 12 months to 24 months. $^{2}$ In my institution, we inform patients that, 3 months to 4 months after surgery, most patients are significantly better than pre-operatively regarding range of motion and level of pain, although it will take a year until they achieve the best possible outcome. Some patients recover very quickly and stop taking analgetics after a few weeks, whereas others continue to use analgetics for several months. As long as gradual improvement is seen, it is most likely a normal postoperative recovery. However, if there is increasing pain or stiffness developing after a period of normal recovery, this could indicate a complication.

Capio St Görans Hospital Stockholm, II2 8I Sweden

Corresponding author:

Anders L. Ekelund, Department of Orthopaedics, Capio St Görans Hospital, SE-II2 8I, Stockholm, Sweden.

Email: anders.ekelund@capiostgoran.se 
Table I. Indications for reverse arthroplasty at the author's institution, 2006-2016 $(n=967)$

\begin{tabular}{|c|c|c|}
\hline Diagnosis & Number of cases & $\%$ of cases \\
\hline Acute proximal humeral fracture & 208 & $21.5 \%$ \\
\hline Cuff arthropathy & 167 & $17.3 \%$ \\
\hline Revision of failed arthroplasty & 165 & $17 \%$ \\
\hline Massive rotator cuff tear without arthritis & 135 & $14 \%$ \\
\hline Malunited fracture & 76 & $7.9 \%$ \\
\hline Osteoarthritis with thin cuff & 72 & $7.4 \%$ \\
\hline Rheumatoid arthritis & 65 & $6.7 \%$ \\
\hline Infected arthroplasty & 28 & $2.9 \%$ \\
\hline Non-union, proximal humerus & 19 & $2 \%$ \\
\hline Miscellanous & 21 & $2.2 \%$ \\
\hline Osteoarthritis with B2- or C-glenoid & II & $1.1 \%$ \\
\hline
\end{tabular}

\section{Analysis and Aetiology of pain}

It is important to determine whether or not there was a trauma before the onset of pain, and whether the pain occurs at rest or when using the arm. Acute pain after a significant trauma can be caused by fractures (scapula, humerus or clavicle), dislocation or rotator cuff injuries. Acute pain can also occur without trauma, and the most common aetiology during the initial postoperative years is a fracture of the acromion or spine of scapula. ${ }^{3-7}$ In a few cases, sudden pain has been caused by spontaneous periprosthetic fractures (Figure 1) and coracoid process fractures. ${ }^{8}$ In these cases, the pain is usually more intense when the patient is trying to use the arm and reduced when the arm is at rest. A gradual increase in pain at rest and stiffness should lead to a high suspicion of infection. ${ }^{9,10}$ Diagnosis of an infection after RSA can be challenging, particularly if the blood tests [erythrocyte sedimentation rate (ESR) and C-reactive protein (CRP)] are normal. ${ }^{11-16}$ Loosening of the prosthetic components can cause pain and loss of function, although aseptic loosening is rare during the initial postoperative years. ${ }^{17-20}$

Location of the pain can guide the surgeon in the diagnosing process. Thus, pain in the proximal part of the upper arm can indicate humeral loosening, spontaneous periprosthetic fracture or infection; pain over the scapula can indicate a fracture of the spine of scapula or suprascapular nerve irritation by the superior screw of the glenoid component. In the author's series, the acromioclavicular (AC) joint has become painful after surgery in a few cases. The pain is usually located over the AC joint and injection of local anaesthesia can confirm the diagnosis.

\section{Diagnosis}

When facing a patient with a painful RSA, it is important to have a structured approach based on the most common complications seen after RSA., ${ }^{7,91-23}$ Table 2 reports the common clinical characteristics of the three most common complications after RSA.

The first step is a careful patient history, which can give valuable information and, in many cases, a preliminary diagnosis that must be confirmed using various diagnostic tools. Physical examination to record passive and active range of motion, strength, atrophies and local tenderness is performed. Standard radiographs are taken and compared with the postoperative images. It is crucial that optimal postoperative images are available. At the author's institution, all postoperative radiographs are taken under fluoroscopic control 2 days after surgery, aiming to achieve optimal views. These images are analyzed regarding signs of implant loosening or dissociation, radiolucencies, infection, dislocation, glenoid notching, fractures of the acromion or spine of scapula, humeral fissure or fracture, base of coracoid fracture, and possible screw problems (Figure 2). It is difficult to diagnose some of these complications on plain radiographs. If the standard radiographs do not give a clear diagnosis, a computed tomography (CT) scan is performed. This is particularly helpful in diagnosing a suspected fracture of the 
(b)
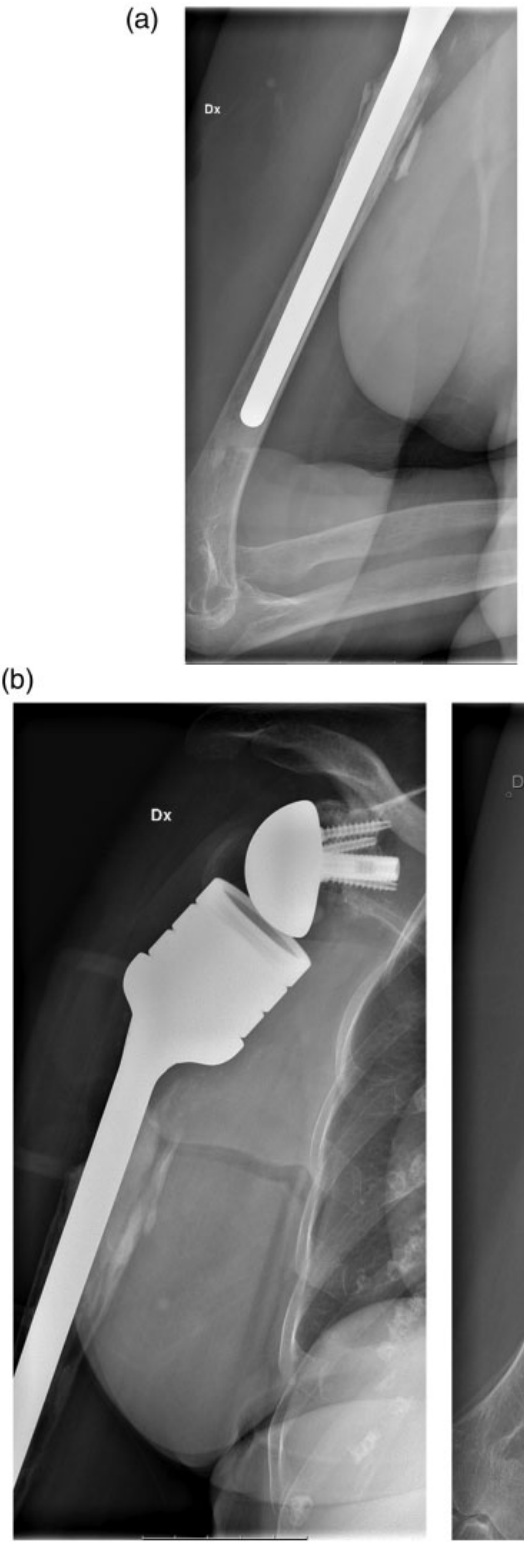

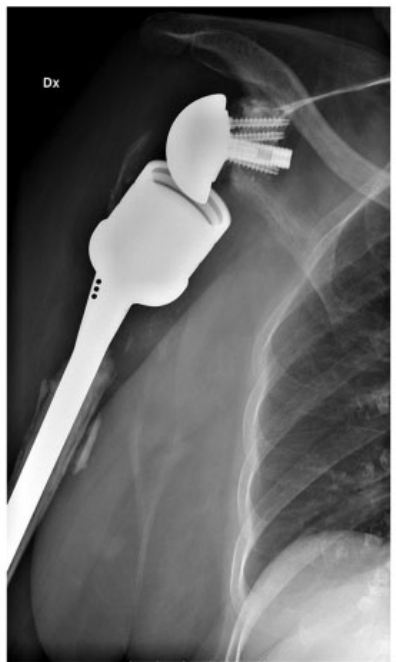

(c)

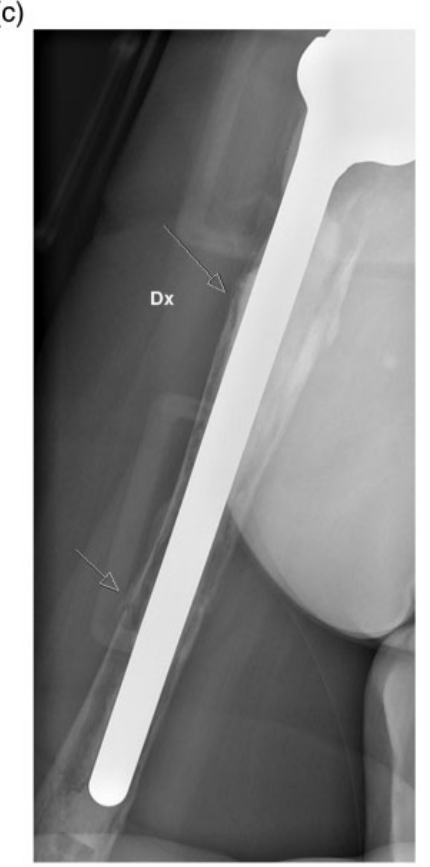

Figure I. (a) Postoperative radiographs after revision of a failed anatomical arthroplasty to a reverse shoulder arthroplasty. (b) Radiographs showing a spontaneous periprosthetic fracture causing intense pain. Treated non-operatively in a brace. (c) Radiograph showing callus formation and clinical improvement.

Table 2. Clinical characteristics of periprosthetic infection, dislocation, and postoperative fractures of the acromion or scapular spine after reverse total shoulder arthroplasty

\begin{tabular}{|lll|}
\hline Pain & Range of motion & Diagnosis \\
\hline Gradual increase in pain at rest & Increasing stiffness & Periprosthetic infection \\
\hline No or limited pain & Sudden loss of active elevation & Dislocation \\
\hline Sudden severe pain & Sudden loss of active elevation & Fracture of scapular spine \\
\hline
\end{tabular}


acromion or scapular spine. Routine blood tests, including leukocytes, ESR and CRP, are performed.

The most common complications reported after RSA are infection, dislocation, glenoid or humeral loosening, fractures of the acromion or spine of scapula, periprosthetic fractures, and glenoid notching. ${ }^{7,9,21-25}$ The author has used the RSA since 1995, and the same system of RSA has been used since 2006 (Delta Xtend; DePuy Synthes, Warsaw, IN, USA). The complications have been reduced compared to the first series of RSAs conducted between 1995 and 2006. The most common complications in the author's series since 2006 (1255 RSA) have been dislocation (1.4\%), acromion and scapular spine fractures $(1.3 \%)$, infection $(1.2 \%)$, and humeral loosening $(0.5 \%)$ (Table 3$)$. Based on the patient history, clinical examination, radiographs, CT scan and blood tests, an attempt should be made to determine the aetiology of the painful RSA. If the

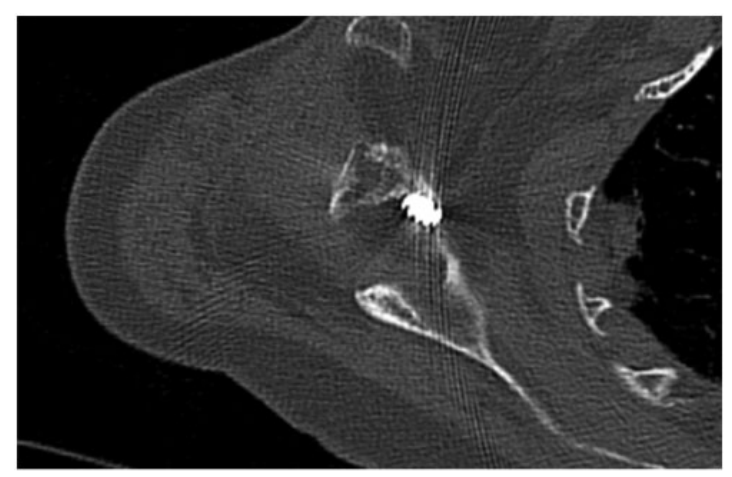

Figure 2. Radiograph showing superior screw that caused irritation of the suprascapular nerve, resulting in pain over the scapula. The screw was removed and the pain disappeared. more common complications have been ruled out, it is important to look for other possible causes of pain, such as lung tumour, referred pain from the neck, protruding cement around the humerus, AC joint pain, and overstretching of the brachial plexus. ${ }^{26,27}$

\section{Instability}

Instability is usually diagnosed during the first year, although late instability can be seen after several years as a result of polyethylene wear and stretching of the soft tissues. ${ }^{7,11,21,28-33}$ At the time of diagnosis, a chronically dislocated RSA is often seen on plain radiographs (Figure 3 ). However, recurrent dislocations or subluxations have also been reported..$^{11,21,29,30,34}$ In the author's series, patients with a chronically dislocated RSA had no or very little pain. The most important symptom was loss of function. However, Black et al. ${ }^{28}$ reported six cases of instability with an average pain score (visual analogue scale) before revision surgery of 6.9. Thus, instability can be very painful. Diagnosis is usually easy. However, the author has seen three cases of painful, stiff RSA where open biopsies were performed to rule out a low-grade infection. The biopsies were negative but, at the time of surgery, there was an instability in the system (increased separation between the components when pulling on the arm). The humeral component was lengthened, with a significant reduction of pain and improved function in these three cases. The patients had not experienced any feeling of instability before revision surgery., we now call this condition, 'painful micro-instability' (Figure 4).

Treatment of instability depends on the causative mechanisms. ${ }^{35}$ In most cases, the instability is caused

Table 3. Complications recorded between 2006 and 2016 in 1259 reverse shoulder arthroplasties: 13 patients had a second complication after the first, and four patients had a third complication

\begin{tabular}{|c|c|c|c|}
\hline Type of complication & $\begin{array}{l}\text { First complication, } \\
n(\%)\end{array}$ & $\begin{array}{l}\text { Second complication, } \\
n(\%)\end{array}$ & $\begin{array}{l}\text { Third } \\
\text { complication, } n(\%)\end{array}$ \\
\hline Instability & $15(1.2)$ & & $2(0.2)$ \\
\hline Postoperative acromion or spine fracture & $14(1.1)$ & $3(0.2)$ & \\
\hline Periprosthetic fracture & II (0.9) & $4(0.3)$ & \\
\hline Infection & $10(0.8)$ & $3(0.2)$ & $2(0.2)$ \\
\hline Loosening of humeral component & $6(0.5)$ & & \\
\hline Loosening of glenoid component & $3(0.2)$ & & \\
\hline Haematoma, needed evacuation & $\mathrm{I}(0.1)$ & & \\
\hline Miscellanous & II (0.9) & $3(0.2)$ & \\
\hline
\end{tabular}


(a)

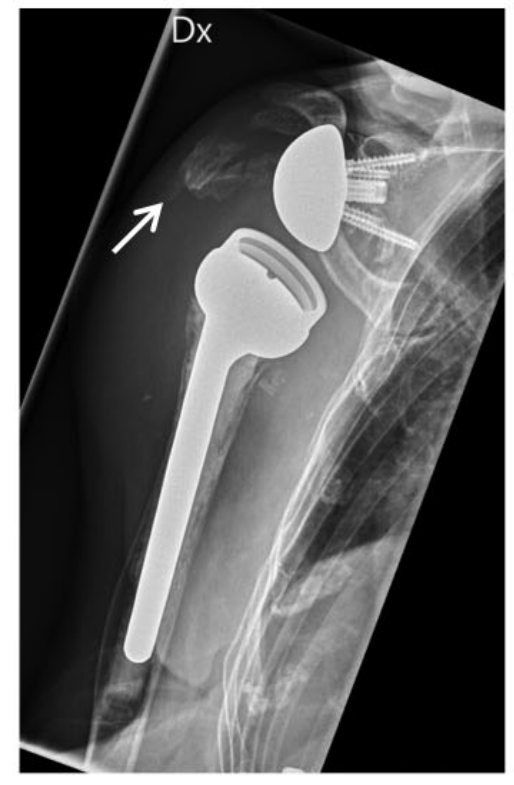

(b)

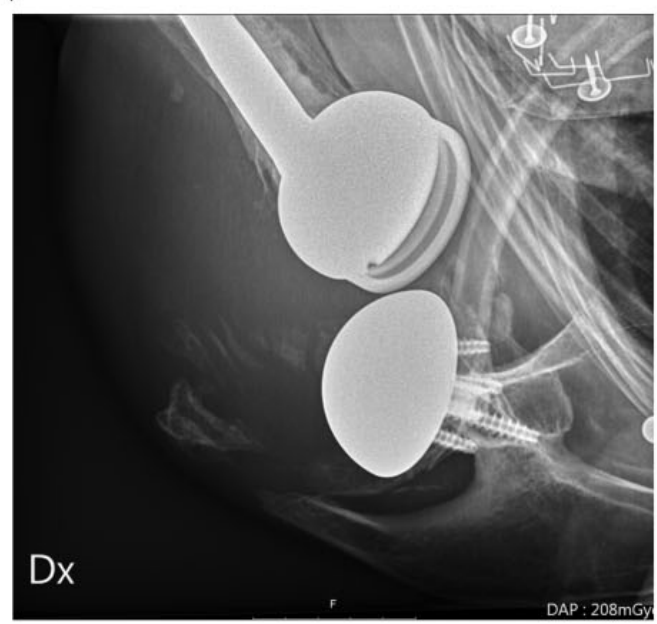

(c)

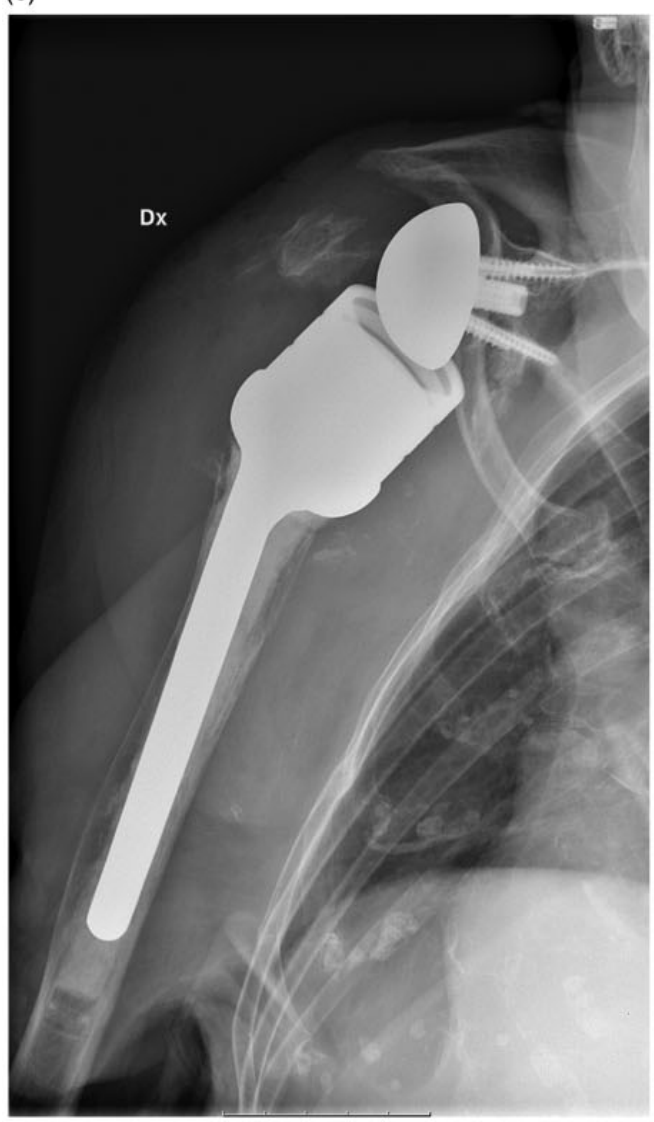

Figure 3. (a, b) Images showing a dislocation of a reverse arthroplasty and a non-union of the scapular spine. (c) The patient was treated by lengthening the humeral component. 
(a)

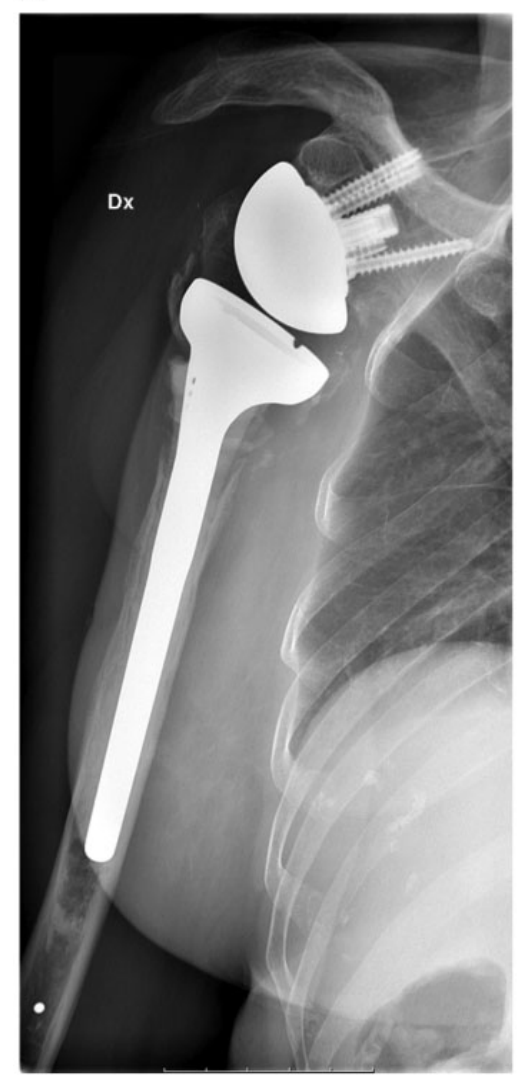

(b)

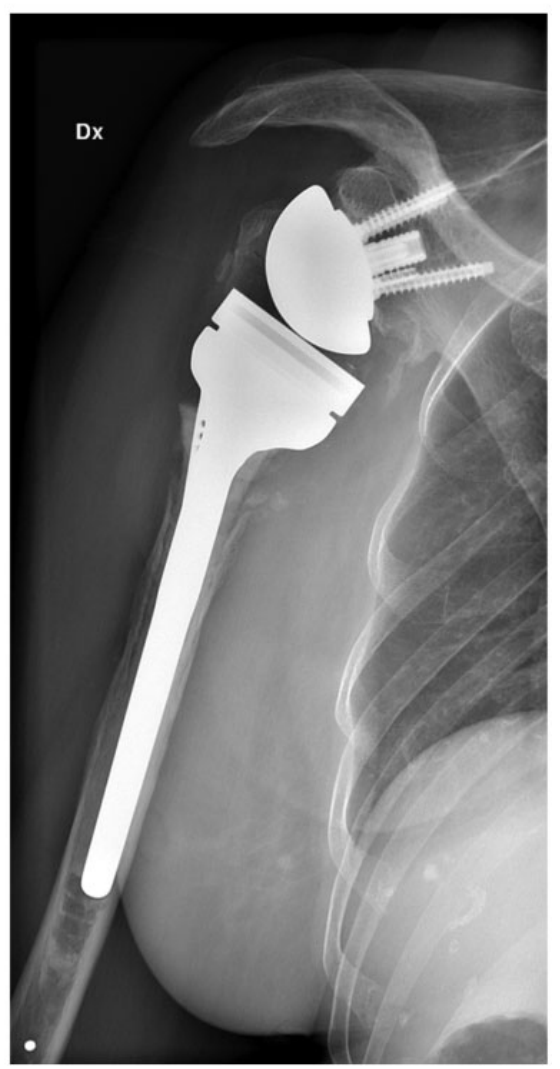

Figure 4. (a) Radiograph of a patient with a painful reverse arthroplasty that was found to have 'micro-instability'. (b) This was treated with lengthening of the humeral component.

by insufficient soft tissue tensioning. Other causes are poor implant poisitioning, impingement, scar tissue (bony fragment) or medialization of the glenosphere (poor deltoid wrapping angle). Instability has also been reported to be associated with infection and postoperative fractures of the scapular spine. ${ }^{11,34}$

Closed or open reduction has been attempted, although the results have been modest. In three reports of revision of failed reverse shoulder arthroplasties, a total of 25 cases of instability were treated with closed or open reduction. ${ }^{29,31,33}$ Seventeen $(68 \%)$ of these failed and needed further surgery. Boileau et al. ${ }^{29}$ had 10 out of 15 cases failing closed or open reduction, Stephens et al. ${ }^{33}$ had five out of five cases failing, and Farshad et al. ${ }^{31}$ had two out of five cases failing. The most common procedure for instability of RSA is release, removal of scar tissue and bony fragments, and lengthening of the humeral component. Boileau et al. $^{29}$ found humeral shortening of more than $15 \mathrm{~mm}$ and medialization of the humeral axis of more than $15 \mathrm{~mm}$ (measured from the border of the acromion) to be significant risk factors for instability. Humeral shortening was treated with metallic spacer or by cementing a humeral component higher. Medialization of the glenoid was treated with a larger glenosphere, a lateralized glenosphere or a bone graft on the glenoid. However, instability remains a challenge. The author has revised 28 cases of instability with an overall success rate of $82 \%{ }^{30}$ Humeral lengthening was performed in 18 patients and, in 10 patients, other procedures were needed. Two patients developed periprosthetic infection. We now consider closed reduction only during the initial postoperative weeks, when the deltoid muscle is still affected by the surgical procedure. After that, instability is treated with revision surgery, most often humeral lengthening.

\section{Infection}

In a recent literature review of complications after reverse arthroplasty, the infection rate was $3.8 \%{ }^{7}$ However, new implants and improved surgical technique have reduced the incidence of infection and, in our unit, it is $1.2 \%$ (1255 RSAs performed in the period 2006 to 2016). Periprosthetic joint infection of the shoulder can have a different clinical picture to 
that of hip and knee replacement. ${ }^{36}$ The patient often has few general signs of infection such as fever. The surgical wound may look normal, and blood tests such as white blood cell count (WBC), ESR and CRP are often normal or only slightly elevated. ${ }^{37}$ Besides staphylococcus, Propionibacterium acnes has been reported as an important pathogen in the shoulder. $^{12,16,38,39}$ Radiographs are rarely helpful because these are usually normal with no signs of loosening. Thus, diagnosing a periprostethic joint infection in the shoulder remains a challenge. ${ }^{36,40,41}$ If radiographs and blood tests are normal, an aspiration can be attempted. ${ }^{36}$ However, many of these infections are 'dry' (i.e. no fluid can be aspirated). In painful RSAs, arthroscopic or open biopsies can be very helpful to rule out a low-grade infection. ${ }^{42}$ The author prefers open biopsies (at least five) taken from around the implants and, at the same time, old permanent sutures are also sent for microbiology. The cultures are kept for a minimum of 2 weeks because $P$. acnes is a slow-growing organism. Recently, serum or synovial levels of interleukin- 6 and $\alpha$-defensin have been studied for diagnosis of a periprosthetic joint infection. ${ }^{13,14}$ Further studies are needed before the value of these tests can be determined.

Early postoperative periprosthetic infections, within the first 4 weeks to 6 weeks, are treated with lavage and exchange of modular parts of the implant (DAIR: debridement, antibiotics and implant retention). ${ }^{15}$ The same approach can be used in acute haematogenous infection. Late infections are treated with complete one- or two-stage revisions. ${ }^{10,15,16,38,43-45}$ The author considers a one-stage revision if the causative organism is known.

Whenever an RSA is performed in a shoulder where previous surgery has been performed (arthroscopically or open), it is important to routinely take at least five biopsies for microbiology. Unexpected positive cultures have been found in as many as $26 \%$ of patients undergoing revision arthroplasty with no clinical signs of infection. . $^{12,39}$

\section{Postoperative acromion and scapular spine fractures}

The reported incidence varies between $1 \%$ and $7 \% .^{3-6}$ These values are probably an underestimate of the true incidence because this complication is often missed and under-reported. Otto et al. ${ }^{6}$ showed that only $79 \%$ of the fractures were identified by blinded reviewers using plain radiographs alone. The fractures can occur spontaneously or after trauma. A careful patient history gives a high suspicion of an acromial or scapular spine fracture. The patient usually has a normal recovery after surgery and usually very good function of the shoulder

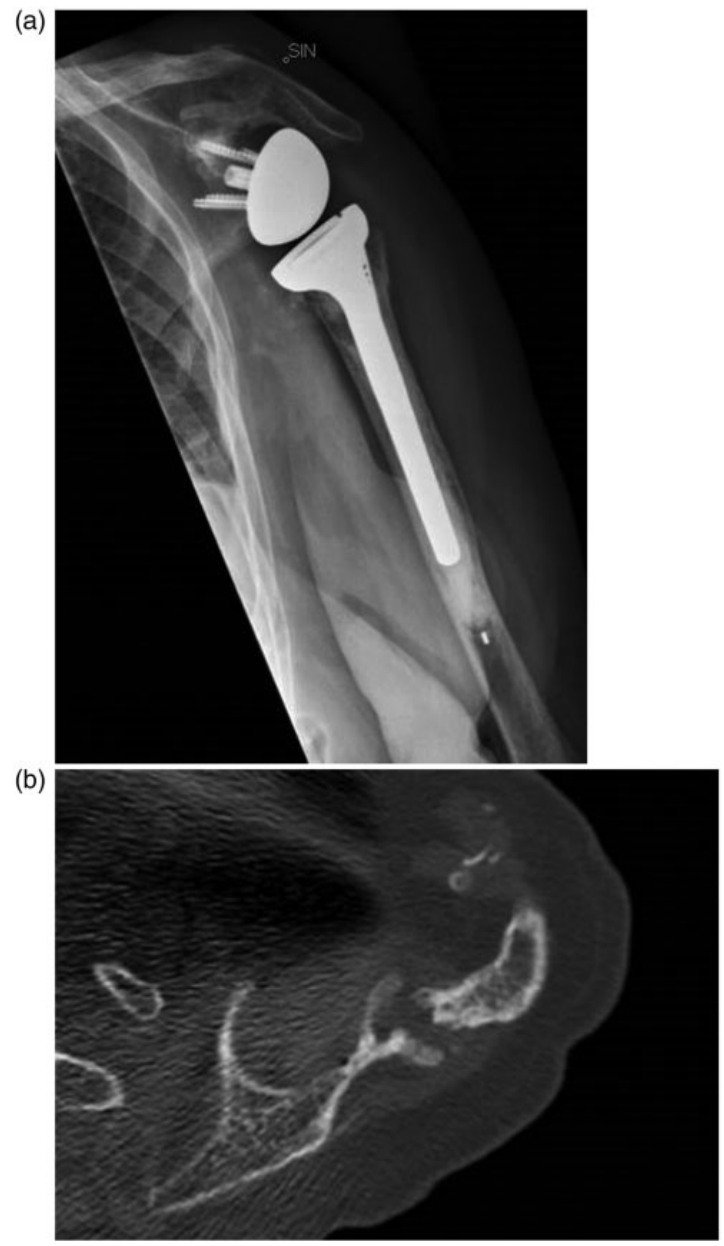

Figure 5. (a) A plain radiograph with the 'tilted acromion sign'. (b) Computed tomography scan with a non-union of the scapular spine.

at the time of fracture. Suddenly, the patient experiences rather severe pain over the shoulder and is unable to lift the arm. The majority of cases occur during the first year, although fractures after many years have been reported. ${ }^{6}$ Physical examination reveals tenderness over the posterior part of the acromion and scapular spine. A plain radiograph can be diagnostic, although any fracture is often missed. Thus, a computed tomography should be performed if the patient history or clinical examination leads to suspicion of a fracture. 'The tilted acromion sign' is often seen on plain radiographs (Figure 5).

These fractures can be treated non-operatively in a sling or abduction brace or by open reduction and internal fixation. Most of the reported fractures have been treated non-operatively. ${ }^{3-6}$ The author considers open reduction and internal fixation in displaced fractures of the scapular spine, although there is currently insufficient literature available to give guidelines regarding the best management of these fractures. 


\section{Spontaneous periprosthetic fracture}

Most periprosthetic fractures occur after a significant trauma. However, a painful RSA can be caused by a fissure or fracture around the implant that happens during activities of daily living (Figure 1). A 74-yearold woman had revision arthroplasty performed. She had significant proximal humeral bone loss. Eleven months after surgery, she reported having increasing pain in the shoulder. Radiographs showed a spontaneous periprosthetic fracture, which was treated in a brace for 10 weeks. Follow-up visits showed callus formation, and the fracture healed. This complication can be missed on plain radiographs, although computed tomography is usually diagnostic. Patients undergoing RSA as a revision procedure, resulting in humeral bone loss, are at risk.

\section{Coracoid base fracture}

Pain over the anterior part of the shoulder around the coracoid process can be caused by a spontaneous fracture at the base of the coracoid. The author has seen one case, which was treated non-operatively in a sling. Anakwenze et al. ${ }^{8}$ recently described two similar cases

(a)

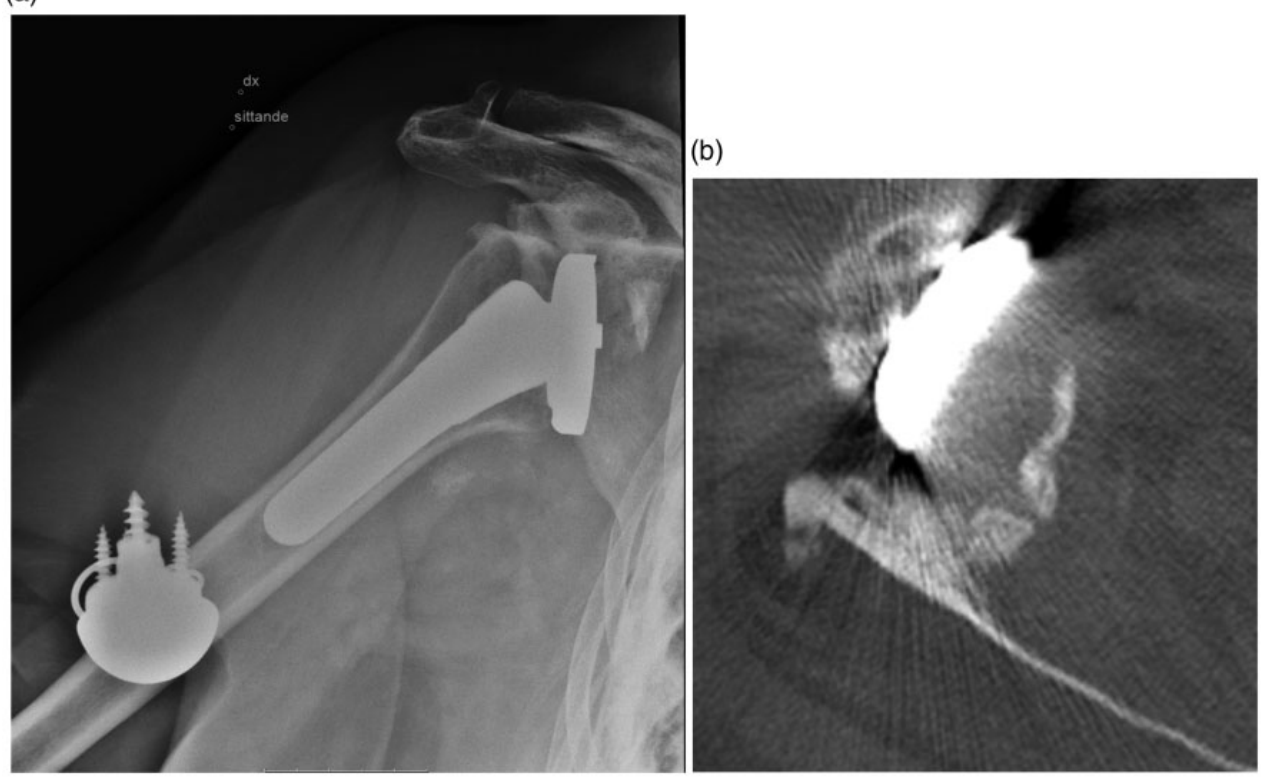

(c)

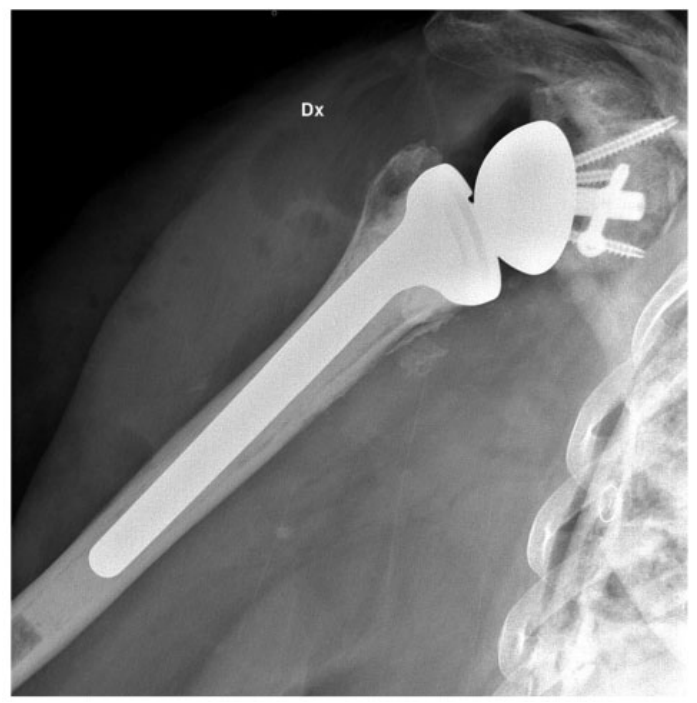

Figure 6. Glenoid component loosening (a) with severe glenoid bone loss (b), treated with glenoid reconstruction with a large bone graft and revision reverse shoulder arthroplasty (c). 
that occurred at 3 months and 15 months after RSA. Computed tomography was diagnostic, and non-operative management resulted in the resolution of symptoms. Coracoid fractures are most likely a result of excess stretch imposed on the conjoined tendon because of lengthening of the arm.

\section{Humeral loosening}

In a review of the literature, Zumstein et al. ${ }^{7}$ found an incidence of humeral loosening of $1.3 \%$. Gilot et al. ${ }^{17}$ reported an incidence of radiographic humeral stem loosening of $0.74 \%$. In the author's series, six cases $(0.5 \%)$ have been revised for this complication. In symptomatic loosening, the patient has pain in the upper part of the arm, particularly when rotation of the shoulder is performed. Plain radiographs and computed tomography can confirm the diagnosis. Prosthetic failure within the first 2 years gives a high degree of suspicion of an infection. ${ }^{20}$ Symptomatic loosening is treated with revision arthroplasty.

\section{Glenoid loosening}

Glenoid loosening after RSA is rare with modern implants and careful surgical technique. ${ }^{7,11,18,22,31}$ The author has revised three cases in a series of 1255 RSAs using modern implants. All three could be successfully revised by inserting a new reverse glenoid component. Holcomb et al. ${ }^{18}$ reported on 14 revisions for failed baseplate in RSA with a lateralized center of rotation design. Revision restored pain relief and function to pre-failure levels. Two patients needed a second revision: one for baseplate failure and one for dislocation. Figure 6 shows a case of glenoid component loosening and its management.

\section{Notching}

Inferior scapular notching is commonly seen after RSA. It has been reported in up to $96 \%$ of cases. ${ }^{7,9,11,24,25,46}$ RSA with a lateralized center of rotation has less notching. The incidence of notching can be affected by the position of the center of rotation, humeral inclination, size of glenosphere, cup depth, downward tilt of the baseplate and position of the baseplate..$^{10,46,47}$ The most important factor is low placement of the baseplate, resulting in an inferior overhang of the glenosphere. ${ }^{46,47}$ Glenoid notching results in bone loss; it is therefore important to try to minimize the risk of notching. However, the clinical impact of glenoid notching is unclear (Figure 7). The author has not performed any revision for glenoid notching since the first RSA was implanted in 1995. In four reports of revision of RSA, with 205 revision procedures in total, none of

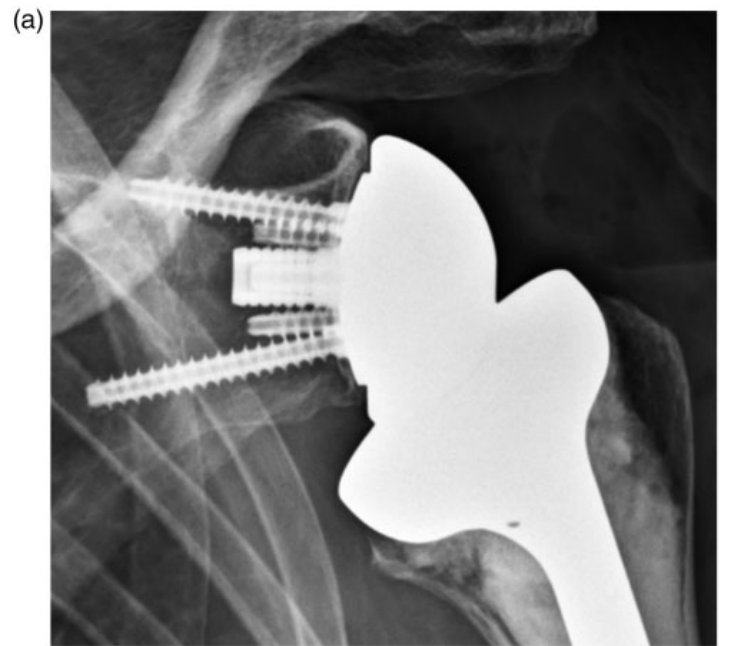

(b)

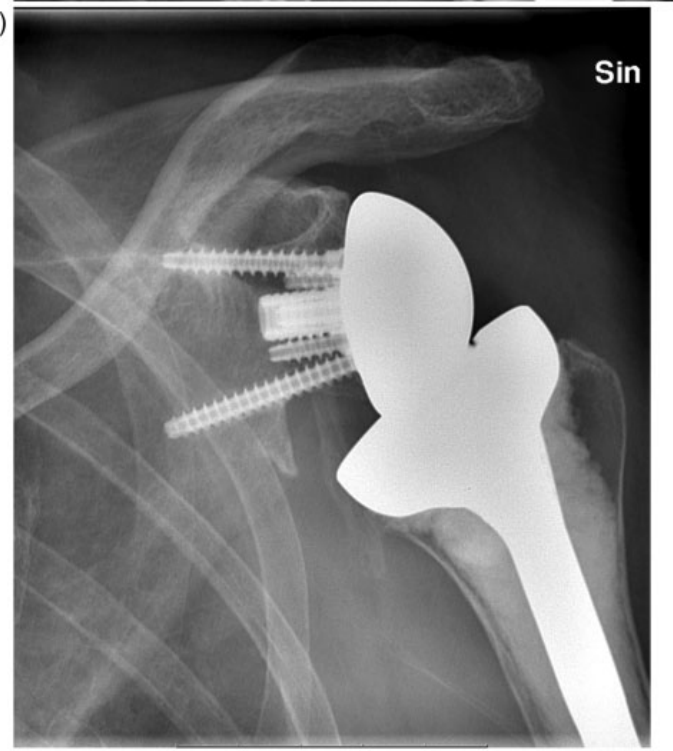

Figure 7. (a) Early postoperative radiograph of a reverse arthroplasty. (b) An image 10 years later, showing asymptomatic inferior glenoid notching.

them were for glenoid notching. ${ }^{28,29,31,33}$ Farshad et $a .^{31}$ reported one case of metallosis as a result of impingement with the inferior screw. The question of whether notching can result in pain and reduced shoulder function remains unanswered.

\section{Suprascapular nerve}

The suprascpaular nerve is at risk when placing the superior screw in the baseplate. The author has seen one case with posterior shoulder pain after RSA, caused by irritation of the suprascpaular nerve in the groove. Computed tomography confirmed the diagnosis (Figure 2). The superior screw was replaced with a shorter screw and the pain disappeared. 


\section{Stretching of the brachial plexus}

The arm is lengthened by $15 \mathrm{~mm}$ to $27 \mathrm{~mm}$ after reverse total shoulder arthroplasty. ${ }^{48}$ Nerve monitoring during shoulder arthroplasties has shown more nerve alerts during RSA than during anatomical total shoulder arthroplasty. ${ }^{49}$ Lenoir et al. ${ }^{50}$ found that the axillary and radial nerves were often stretched during the procedure. The author has seen several cases of postoperative numbness in the hand, particularly in the ulnar nerve area. However, it is unclear whether this can cause postoperative pain in the shoulder and arm. Further research is needed.

\section{Summary}

Reverse total shoulder arthroplasty can relieve pain and restore function in many different shoulder conditions. However, painful complications can occur and it is important to try to determine the cause of pain because many of these complications can be treated successfully. During the first year, infection, postoperative fracture of the acromion or scapular spine and dislocation are the most common reasons for pain. A careful patient history, clinical examination, standard radiographs and routine blood tests (ESR, CRP and WBC) are the first steps in evaluation of a painful RSA. Computed tomography can add valuable information, although magnetic resonance imaging is rarely used by the author. The challenge is to diagnose a lowgrade infection as the source of pain, and aspiration combined with arthroscopic or open biopsy may be necessary. In most cases, the aetiology of pain can be determined and it can be treated, although there is a group of patients with unexplained pain and stiffness.

\section{Declaration of Conflicting Interests}

The author(s) declared no potential conflicts of interest with respect to the research, authorship, and/or publication of this article. The paper has not been presented at any society or meeting.

\section{Funding}

The author(s) received no financial support for the research, authorship, and/or publication of this article.

\section{References}

1. Levy JC, Everding NG, Gil CC, Stephens S and Giveans MR. Speed of recovery after shoulder arthroplasty: a comparison of reverse and anatomical total shoulder arthroplasty. J Shoulder Elbow Surg 2014; 23: 1872-1881.

2. Simovitch R, Flurin P-H, Marczuk Y, et al. Rate of improvement in clinical outcomes with anatomic and reverse total shoulder arthroplasty. Bull Hosp Joint Dis 2015; 1: S111-S117.
3. Crosby LA, Hamilton A and Twiss T. Scapula fractures after reverse total shoulder arthroplasty: Classification and treatment. Clin Orthop Rel Res 2011; 469: 2544-2549.

4. Levy JC and Blum S. Postoperative acromion base fracture resulting in subsequent instability of reverse shoulder replacement. J Shoulder Elbow Surg 2012; 21: e14-e18.

5. Mayne IP, Bell SN, Wright W and Coghlan JA. Acromial and scapular spine fractures after reverse total shoulder arthroplasty. Shoulder Elbow 2016; 8: 90-100.

6. Otto RJ, Virani NA, Levy JC, Nigro PT, Cuff DJ and Frankle MA. Scapular fractures after reverse shoulder arthroplasty: evaluation of risk factors and the reliability of a proposed classification. J Shoulder Elbow Surg 2013; 22: $1514-1521$.

7. Zumstein MA, Pinedo M, Old J and Boileau P. Problems, complications, reoperations, and revisions in reverse total shoulder arthroplasty: a systematic review. J Shoulder Elbow Surg 2011; 20: 146-157.

8. Anakwenze OA, Kanchera VK, Carolan GF and Abboud J. Coracoid fracture after reverse totsal shoulder arthroplasty: a report of 2 cases. Am J Orthop 2015; 44: E469-E472.

9. Cheung E, Willis M, Walker M, Clark R and Frankle MA. Complications in reverse total shoulder arthroplasty. J Am Acad Orthop Surg 2011; 19: 439-449.

10. Coste JS, Reig S, Trojani C, Berg M, Walch G and Boileau P. The management of infection i arthroplasty of the shoulder. J Bone Joint Surg 2004; 86B: 65-69.

11. Farshad M and Gerber C. Reverse total shoulder arthroplasty - from the most to the least common complication. Int Orthop (SICOT) 2010; 34: 1075-1082.

12. Foruria AM, Fox TJ, Sperling JW and Cofield RH. Clinical meaning of unexpected positive cultures (UPC) in revision shoulder arthroplasty. J Shoulder Elbow Surg 2013; 22: 620-627.

13. Frangiamore SDJ, Saleh A, Grosso MJ, et al. Alphadefensin as a predictor of periprosthetic shoulder infection. J Shoulder Elbow Surg 2015; 24: 1021-1027.

14. Frangiamore SJ, Saleh A, Kovac MF, et al. Synovial fluid interleukin-6 as a predictor of periprosthetic shoulder infection. J Bone Joint Surg 2015; 97A: 64-70.

15. Kuiper JWP, Willink RT, Moojen DJF and van den Bekerom MPJ. Treatment of acute periprosthetic infections with prosthesis retention: review of current concepts. World J Orthop 2014; 18: 667-676.

16. Ortmeier R, Resch H, Hitzl W, Mayer M, Stundner O and Tauber M. Treatment strategies for infection after reverse shoulder arthroplasty. Eur J Orthop Surg Traumatol 2014; 24: 723-731.

17. Gilot G, Alvafrez-Pinzon AM, Wright TW, et al. The incidence of radiographic aseptic loosening of the humeral component in reverse total shoulder arthroplasty. $J$ Shoulder Elbow Surg 2015; 24: 1555-1559.

18. Holcomb JO, Cuff D, Petersen SA, Pupello DR and Frankle MA. Revison reverse shoulder arthroplasty for glenoid baseplate failure after primary reverse shoulder arthroplasty. J Shoulder Elbow Surg 2009; 18: 717-723.

19. Phadis J, Huang T, Watts A, Krishnan J and Bain GI. Cemented or cementless humeral fixsation in reverse total 
shoulder arthroplasty? Bone Joint Journal 2016; 98B: 65-73.

20. Portillo ME, Salvado M, Alier A, et al. Prosthesis failure within 2 years of implantation is highly predictive of infection. Clin Orthop Rel Res 2013; 471: 3672-3678.

21. Clark JC, Ritchie J, Song FS, et al. Complication rates, dislocation, pain, and postoperative range of motion after reverse shoulder arthroplasty in patients with or without repair of the subscapularis. J Shoulder Elbow Surg 2012; 21: $36-41$.

22. Scarlat MM. Complications with reverse total shoulder arthroplasty and recent evolutions. International Orthop (SICOT) 2013; 37: 843-851.

23. Wierks C, Skolasky RL, Ji JH and McFarland EG. Reverse total shoulder arthroplasty. Clin Orthop Rel Res 2009; 467: 225-234.

24. Levigne C, Boileau P, Favard L, et al. Scapular notching in reverse shoulder arthroplasty. J Shoulder Elbow Surg 2008; 17: 925-935.

25. Levigne C, Garret J, Boileau P, Alami G, Favard L and Walch G. Scapular notching in reverse shoulder arthroplasty: is it important to avoid it and how? Clin Orthop Rel Res 2011; 469: 2512-2520.

26. Lädermann A, Stimec BV, Denard PJ, Cunningham G, Collin $\mathrm{P}$ and Fasel JH. Injury to the axillary nerve after reverse shoulder arthroplasty: an anatomical study. Orthop Traumatol Surg Res 2014; 100: 105-108.

27. Marion B, Leclère FM, Casoli V, et al. Potential axillary nerve stretching during RSA implantation: an anatomical study. Anat Sci Int 2014; 89: 232-237.

28. Black AEM, Roberts SM, Siegel E, Yannopoulos P, Higgins LD and Warner JP. Failure after reverse total shoulder arthroplasty: what is the success of component revision? J Shoulder Elbow Surg 2015; 24: 1908-1914.

29. Boileau P, Melis B, Duperron D, Moineau G, Rumain AP and Han Y. Revision surgery of reverse shoulder arthroplasty. J Shoulder Elbow Surg 2013; 22: 1359-1370.

30. Ekelund A. Instability after reverse arthroplasty. In: Walch G, Boileau P, Molé D, Favard L, Lévigne C, Sirveaux F, eds. Complications in shoulder arthroplasty. Montpellier: Saurams Medical, 2012.

31. Farshad M, Grögli M, Catanzaro S and Gerber C. Revision of reversed total shoulder arthroplasty: indications and outcome. MBC Musculoskel Disord 2012; 13: 160.

32. Gallo RA, Gamradt SC, Mattern CJ, et al. Instability after reverse total shoulder arthroplasty. J Shoulder Elbow Surg 2011; 20: 584-590.

33. Stephens BC, Simon P, Clark ARE, et al. Revision for failed reverse: a 12-year review of a lateralized implant. J Shoulder Elbow Surg 2016; 25: e115-e124.

34. Trappey GJ, O'Connor DP and Edwards TB. What are the instability and infection rates after reverse shoulder arthroplasty. Clin Orthop Rel Res 2011; 469: 2505-2511.

35. Gutierrez S, Keller TS, Levy JC, Lee WE and Luo ZP. Hierarchy of stability factors in reverse shoulder arthroplasty. Clin Orthop Rel Res 2008; 466: 670-676.

36. Ricchetti ET, Frangiamore SJ, Grosso MJ, Alolabi B, Saleh A, Bauer TW and Ianotti JP. Diagnosis of periprosthetic infection after shoulder arthroplasty - a critical analysis review. J Bone Joint Surg Rev 2013; 1: 1.

37. Berbari E, Mabry T, Tsaras G, et al. Inflammatory blood laboratory levels as markers of prosthetic joint infection: a systematic review and meta-analysis. J Bone Joint Surg 2010; 92: 2102-2109.

38. Sabesan VJ, Ho JC, Kovacevic D and Ianotti JP. Twostage reimplantation for treating prosthetic shoulder infection. Clin Orfthop Rel Res 2011; 469: 2538-2543.

39. Topolski MS, Chin PYK, Sperling JW and Cofield RH. Revision shoulder arthroplasty with positive intraoperative cultures: the value of preoperative studies and intraoperative histology. J Shoulder Elbow Surg 2006; 15: 402-406.

40. The Workgroup Convened by the Musculoskeletal Infection Society. New definition for periprosthetic joint infection. J Arthroplasty 2011; 26: 1136-1138.

41. Updegrove GF, Armstrong AD and Kim HMM. Preoperative and intraoperative infection workup in apparently aseptic revision shoulder arthroplasty. J Shoulder Elbow Surg 2015; 24: 491-500.

42. Dilisio MF, Miller LR, Warner JJP and Higgins LD. Arthroscopic tissue culture for the evaluation of periprosthetic shoulder infection. J Bone Joint Surg 2014; 96A: 1952-1958.

43. Beekman PDA, Katusic D, Berghs BM, Karelse A and De Wilde L. One-stage revision for patients with a chronically infected reverse total shoulder replacement. $J$ Bone Joint Surg 2010; 92B: 817-822.

44. Grosso MJ, Sabesan VJ, Ho JC, Ricchetti ET and Ianotti JP. Reinfection rates after 1-stage revision shoulder arthroplasty for patients with unexpected intraoperative cultures. J Shoulder Elbow Surg 2012; 21: 754-758.

45. Zavala JA, Clark JC, Kissenberth MJ, Tolan SJ and Hawkins RJ. Management of deep infection after reverse total shoulder arthroplasty: a case series. J Shoulder Elbow Surg 2012; 21: 1310-1315.

46. Simovitch RW, Zumstein MA, Lohri E, Helmy N and Gerber C. Predictors of scapular notching in patients managed with the Delta III reverse shoulder arthroplasty. J Bone Joint Surg 2007; 89: 588-600.

47. De Wilde LF, Poncet D, Middernacht B and Ekelund A. Prosthetic overhang is the most effective way to prevent scapular conflict in reverse total shoulder arthroplasty. Acta Orthop 2010; 81: 719-726.

48. Lädermann A, Edwards TB and Walch G. Arm lengthening after reverse shoulder arthroplasty: a review. International Orthop (SICOT) 2014; 38: 991-1000.

49. Parisien RL, Yi PH, Hou L, Li X and Jawa A. The risk of nerve injury during anatomical and reverse total shoulder arthroplasty: an intraoperative neuromonitoring study. J Shoulder Elbow Surg 2016; 25: $1122-1127$.

50. Lenoir H, Dagneaux L, Canovas F, Waitzenegger T, Pham TT and Chammas M. Nerve stress during reverse total shoulder arthroplasty: a cadaveric study. J Shoulder Elbow Surg 2017; 26: 323-330. 